\author{
Proceedings of the $10^{\text {th }}$ International Conference on Applied Informatics \\ Eger, Hungary, January 30-February 1, 2017. pp. 163-170 \\ doi: $10.14794 /$ ICAI.10.2017.163
}

\title{
Spreading models on moving agents
}

\author{
Gergely Kocsis \\ University of Debrecen, Faculty of Informatics \\ kocsis.gergely@inf.unideb.hu
}

\begin{abstract}
In this work we investigate different spreading models on dynamic topogies of agents. Agents of the system take place on a regular square lattice and they are moving as time passes. It was shown previously [1] that varying the dynamics of the agents results in topological changes if we examine the system in discrete time steps. Namely for low speed the network of agents stays close to regular lattices. Increasing the speed (the distance the agents jump in each time-step) brings the topology to small-world, while in some cases even scale-free like topologies can be seen. In our work we use SI model variants [2] to test if there is any difference between spreading processes on static topologies and the same processes on dynamic topologies introduced above.
\end{abstract}

Keywords: spreading, agent-based models, SI model, topologies

$M S C: 68$

\section{Motivation}

Spreading (diffusion) phenomena is in the focus of research for a couple of decades $[3,4]$. Studying the literature of the topic soon reveals that the underlying topology may affect the behavior of the spreading model. More precisely the more long-range connections are present in the network, the more the spreading process speeds up [5]. Most of these early works however focus only on the diffusion processes on static networks. The assumption that during the spreading process the network keeps more or less unchanged may be true in many cases (e.g. simple forest fire, information spreading between companies, broadcasting on a local computer network). However there are many cases where this does not hold [6]. E.g. for disease spreading one of the key roles of spreading the disease all around the society is that the agents (the people of the society) are moving from one place to another. In this current work we investigate how spatially dynamic systems can be modeled using dynamic topologies. More precisely we apply SIS dynamics on topologies inspired by molecular dynamics [2, 7]. Our study builds up from two steps: i.) provide a 
model and dynamics for the network and ii.) provide a model for the spreading. We predict that by varying the agent density and dynamics we arrive to different topologies at given points of time (regular lattice, random network) while if we introduce more complicated agent dynamics even complex network topologies can be achieved. These topologies may highly affect the spreading [7]. Since the work is still in its early stage we investigate only one SIS model variant on the resulted networks, but still we introduce some interesting results.

\section{Model and dynamics}

\subsection{Agents' network dynamics}

As it was mentioned above our model's dynamics build up from two parts from which the first one is the dynamics of the network topology based on the movement of agents. As a basic starting point we use a model inspired by the work of Peruani and Sibona [7]. Namely we put $N$ number of agents on a square lattice of side length $L$. This implies that we have a system where $p=N /(L x L)$ percentage of the available places are occupied by agents. Agents on the network can change their place in discrete time steps using sequential update in order to avoid collisions of agents or having multiple agents on one cell [8]. One time step means $N$ agent movements, where agents are selected randomly (Note that this implies that there may be agents moving more than once in a time step while others may not move at all.). The "social" network of agents builds up by defining edges between those agents that are on neighboring cells after the time step. In order to model real world phenomena more precisely we sophisticate the building dynamics by introducing a memory of agents meaning that agents do not forget their previous neighbors in each step but they remember all the agents they have contacted in the past $m$ steps, where $m$ is a whole number that is the same for all agents. In our model the control parameter of the dynamics is the density $p$ of agents. This way we can also use the contact rate of agents meaning the amount of agents met in a given interval of time. This clearly depends on the density of the agents and the selected size of the time interval.

What we have changed in the dynamics is thee way how agents move on the lattice. While in the original paper simple molecular dynamics rules were applied we found that not really realistic (it is hard to find examples of social systems, where agents are moving like gas particles). Our suggestion is to change this dynamics to more realistic ones.

Brownian dynamics Our first proposed model of agent movement dynamics was inspired by Brownian motion [9]. This easy to implement rule set means that in each time step the agents may move to a neighboring cell on the lattice selected randomly from the free cells out of the eight neighborings of the agent. If all the neighboring cells are occupied the agent stays at its original place. After this we define that an agent is a neighbor of another if they are at neighboring places after 
the time step. This process is repeated as many times as the value of the memory of agents $m$.

Levy dynamics It was shown that in nature in many cases agents of societies move following more sophisticated rules than what Brownian motion can provide. Namely the movement trajectories of these agents can be described by the so called Levy-flight dynamics [10]. This means that in most of the time agents are moving close to their actual position randomly but in some few cases they make huge jumps. (Think of hordes of animals for example moving around the same place in a day but making long journeys sometimes yearly, or think of people going to work and back home each day, but sometimes go for holiday on weekends to very far places.) In order to implement these dynamics we updated the network building rules in the following way: The selected agent can move to any other free positions on the lattice, however the probability of choosing a cell is decreasing following a power law function of the distance. More precisely the probability of choosing a cell that is $\delta$ cells far from the actual position is $q(\delta)=\delta^{-1}$ [11]. The direction is selected randomly. In this way the agent chooses a place to move. If it is not empty it tries another one. After a limit of 100 trials the agent gives it up and stays at its current position. Further extensions of the model may include a non-constant density dependent number for this limit.

Preferential movement dynamics In network science preferential attachment network building dynamics is very well-known and in fashion in the past two decades [12]. The advantage of it is that by using it one can generate network topologies with degree distributions following a power law form. These networks are often called scale-free networks and examples of their presence in the real world has been shown many times in the literature [12]. In order to be able to reproduce the properties of these networks (many agents having only a few neighbors and only some having really high degree) we introduced a new type of agent movement dynamics that we named "preferential movement" dynamics. In this case agents are selected to move proportionally to how many times they have been moved before. This means that at the first step all agents have the same probability to move, but next time the one that has already moved has a bigger chance to move again. After selecting an agent for simplicity it moves following the simple above mentioned Brownian movement dynamics. Note that "preferential movement" only affects the selection of the agents so other movement dynamics could also be applied when moving (e.g. Levy-flight). From the implementation point of view in this case we set a weight for each agent at the initial step. This weight is 1 for each agent. The probability of selecting an agent is proportional to its weight. When an agent is selected the weight of it is increased by a constant number $w_{c}$. This implies that next time it has more chance to be chosen than the others. In our later simulations $w_{c}=N / 1000$. 


\subsection{Spreading dynamics}

After being able to generate a dynamic network topology the next step is to define the dynamics of the used spreading model. For this we used a simple SIS (Susceptible-Infected-Susceptible) rule model [2], meaning that if a susceptible agent meets an infected one it also gets infected for a while (for simplicity in our model for one time step) and then turns back to susceptible again. It is important to emphasize that from the point of the spreading model a time step is not the same as from the point of the network building model. Namely we say, that one step of time from the spreading model is as long as the memory of the moving agents. This implies that for e.g. if the memory length is $m=5$ an infected agent infects all the susceptible agents it met among 5 updates of the system.

\section{Results}

\subsection{The resulted networks}

In order to get a basic insight to the structure of the network on Fig. 1 we plotted the degree distribution of the resulted networks for different densities using different memory lengths for agents.
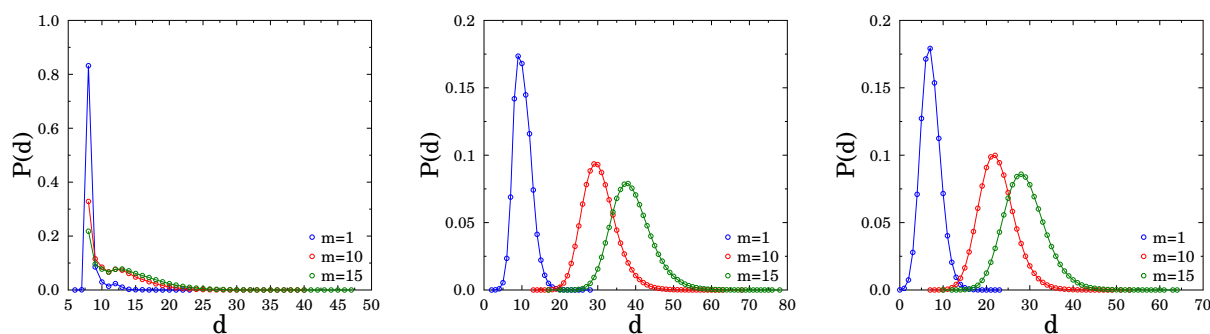

Figure 1: Degree distributions of networks generated using Brownian dynamics with different densities $p$ and memory lengths $m$. left: $p=0.99$, middle: $p=0.75$, right: $p=0.5$. Note the increase of the average degree with the increase of the memory size $m$ and also the results of different densities.

One may note that in general increasing the memory length of agents increase the average degree of nodes of the resulted network. This is not surprising since with longer memory agents have more time to meet others during their dynamics. On the other hand however changing the density of agents results in non-trivial changes in the degree distribution. It is clear that for high densities (Fig. 1 left $p=0.99$ ) almost all agent has the same degree about 8 since they hardly have any place to move no matter how much time they have. Decreasing the density to $p=0.75$ (Fig. 1 middle) results in higher average degrees, but what is interesting 
is that if we keep decreasing the density (Fig. 1 right $p=0.5$ ) the average degree starts to decrease again. The reason of this is that the system has reached a point where the more space for movements does not provide as much advantages for making connections as much the low density hinders it.

Fig. 2 shows the degree distributions for the resulted networks of Levy dynamics. Again the same densities ( $p=0.99, p=0.75$ and $p=0.5$ from left to right) were used as in the case of Brownian dynamics. Note that this change makes an increase of the average degree (not counting the trivial case when the density is almost $p=1$ and the memory length is $m=1$ ). The increase and decrease of averages with the density remains as it was for the Brownian case. What may also be noted that in this case the degree distributions are much wider than in the Brownian case, but this is also a result of the increased number of options of agents when moving.
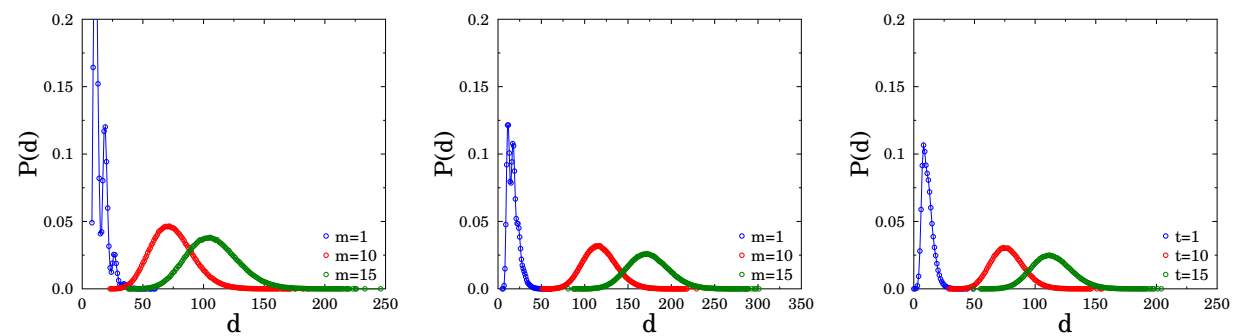

Figure 2: Degree distributions of networks generated using Levyflight dynamics with different densities $p$ and memory lengths $m$. left: $p=0.99$, middle: $p=0.75$, right: $p=0.5$. Note again the increase of the average degree with the increase of the memory size $m$ and also the results of different densities.

In the case of "preferential movement" network generation dynamics as a result we have achieved the previously predicted scale-free topology networks indicated by their degree distributions following a power-law form. Naturally a power-law form cannot be reached for all the cases. If the density $p$ is too high agents barely have any place to move no matter how many times are they selected so a simple almost constant degree appears (Fig. 4 left). For lower densities a power-law part appears in the distribution (Fig. 4 middle) that gets even longer if we lower the density more (Fig. 4 right). Note that seemingly the distributions have an exponential cutoff that seems to loose its importance with the decreasing of the density. In our simulations we found that relatively high values of the constant $\left(w_{c}=\frac{N}{1000}\right.$ on Fig. 3) are needed to get good quality power law degree distributions.

Fig. 4 presents some first results of running spreading simulations on the generated networks. From left to right the subfigures are for Brownian, Levy and preferential movement dynamics. The different curves are for different densities $p$ of agents on the network. In order to separate consequences of different parameters we set the starting infection level in the system to $P(I)_{t=0}=0.1$. Our results show 

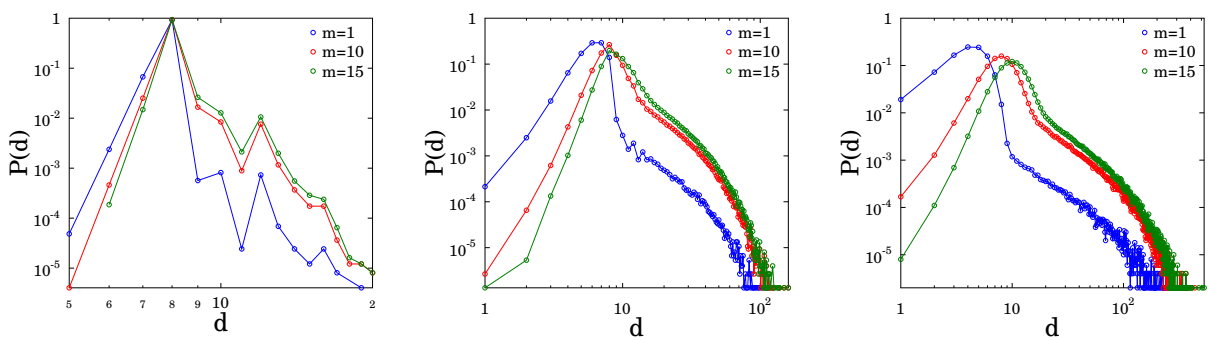

Figure 3: Degree distributions of networks generated using "preferential movement" dynamics with different densities $p$ and memory lengths $m$. left: $p=0.99$, middle: $p=0.75$, right: $p=0.5$. With the right setting of the above parameters the power-law form degree distribution can be achieved.

that independently of the topology and the density the system always converges to a constant state. The above properties however highly effect where is this constant level of infection. Comparing the Brownian dynamics case with the Levy dynamics case (Fig. 4 left and middle) one may note that the long range connections in the system (resulted by the jumps of agents on the lattice) facilitate the spreading so a much lower density is needed to reach the same final level of infection. Comparing the simple Brownian dynamics case with the preferential movement case (Fig. 4 left and right) one can also note that this change of the dynamics (and the resulted network topology) also facilitates the spreading however for the same model parameters the effect is less strong as for the Levy case. Also note that in the simple Brownian case the speed of reaching the final state is slower than in the other two cases. This is of course again may be the result of the more regular topology.
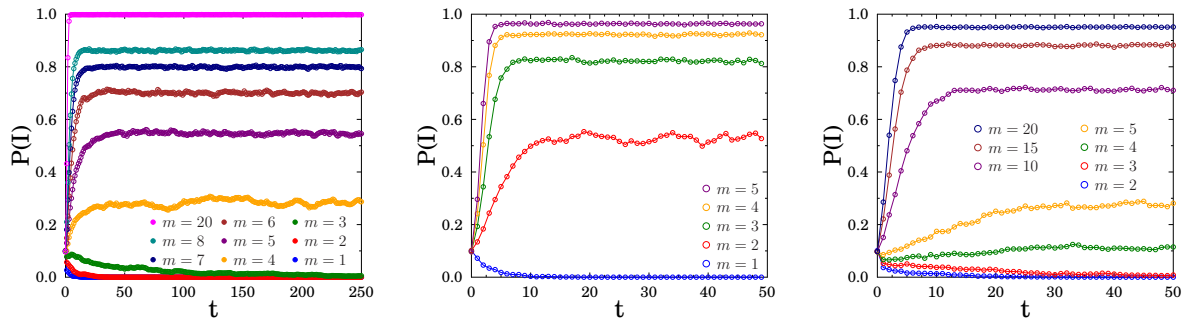

Figure 4: The level of "infection" in the system as a time for different generated network topologies. (left Brownian dynamics, middle Levy dynamics, right "preferential movement" dynamics). 


\section{Discussion}

In this work we investigated spreading models on dynamic topologies of agents. We presented a network generation method inspired by phenomena of statistical physics. We found that changing the networks building dynamics we can produce networks of different types. Through the study of the degree distribution of the networks we have shown that by increasing the agent density on our lattice we can generate qualitatively similar networks but with different average degrees. Even though this method seems to be very promising at the current level we left many questions open so further study of the effect of some parameters are planned in the future. On the generated networks we run simulations by a spreading model of SIS dynamics. We found that in all cases the system converges to an almost fix level of infection. This level depends on the topology of the network and also on the memory length of agents. Just as in the case of the network generation dynamics in this case also other properties may be studied. Also the analytical analysis of the model seems to be feasible with some effort so it is also planned for the future.

\section{References}

[1] J. TAng et. AL., Small-world behavior in time-varying graphs, Physical Review E $81,055101(\mathrm{R})(2010)$.

[2] M. E. J. Newman, Networks - An introduction, University of Michigan and Santa Fe Institute, pp. 627-675, Oxford University Press (2010).

[3] E. M. Rogers, Diffusion of Innovations, Free Press, New York, ISBN: 0-7432-2209-1, (2003).

[4] W. Weidlich, Sociodynamics - A Systematic Approach to Mathematical Modelling in the Social Sciences, Dover Publications Inc., Mineola, New York, ISBN: 0-48645027-9, (2000).

[5] Imre VARga, Comparison of Network Topologies by Simulation of Advertising, In Proceedings of the 2nd International Conference on Complexity, Future Information Systems and Risk (COMPLEXIS 2017), pp. 17-22 ISBN: 978-989-758-244-8 (2017).

[6] László Gulyás, George Kampis, Spreading processes on dynamically changing contact networks, Eur. Phys. J. Special Topics 222 pp. 1359-1376 (2013).

[7] Fernando Peruani and Gustavo J. Sibona, Dynamics and Steady States in Excitable Mobile Agent Systems, PRL 100, 168103 (2008).

[8] Schönfisch B. And de Roos A., Synchronous and asynchronous updating in cellular automata, BioSystems, 51 pp. 123-143 (1999).

[9] Richard Feynman, The Feynman Lectures on Physics Vol I. Addison Wesley Longman. ISBN 978-0-201-02115-8, (1970).

[10] G. Li, S. D. S. Reis, A. A. Moreira, S. Havlin, H. E. Stanley, and J. S. Andrade, JR., Towards Design Principles for Optimal Transport Networks, PRL 104, 018701 (2010). 
[11] Mandelbrot, Benoit B., The Fractal Geometry of Nature, New York: W. H. Freeman. ISBN 0-7167-1186-9. OCLC 7876824 (1982).

[12] Albert-László Barabási, Network Scence, Cambridge University Press, ISBN: 978-1-107-07626-6, (2016). 